\title{
Does GSS Still Maintain Relevance on HAART Outcome After the Introduction of Newest Active Antiretroviral Drugs? 48 Weeks Results
}

\author{
M. Ortu ${ }^{*}, 1$, P. Vitiello ${ }^{1}$, F. Adorni ${ }^{2}$, R. Rossotti ${ }^{1,}$, P. Di Vincenzo ${ }^{1,}$, O. Viganó ${ }^{1}$, M. Galli ${ }^{1}$ and \\ S. Rusconi ${ }^{*}, 1$ \\ ${ }^{I}$ Clinical Sciences Department “Luigi Sacco”, Infectious Diseases and Immunopathology Section, University of Milan, \\ Italy; ${ }^{2} I T B-C N R$, Segrate (MI), Italy
}

\begin{abstract}
Background: Since recent observations demonstrated that extended resistance to protease inhibitors, nucleosidic and non - nucleosidic retrotranscriptase inhibitors (PI, NRTI, NNRTI) is a marker of disease progression and death, it is a matter of the greatest importance that experienced human immunodeficiency virus (HIV) - infected patients with limited therapeutic options receive a suppressive therapy pending the availability of at least two new antiretroviral drugs. Aim of the present study is to evaluate if the GSS score, calculated by analyzing the resistance to historical antiretroviral drugs and drug classes, is still relevant since several new potent drugs and drug classes entered the current clinical use.
\end{abstract}

Methods: Taking into account patients without suppression of HIV replication for $\geq 6$ months from October 2008 and October 2009, we analyzed viroimmunological and resistance data of 38 outpatients starting their last antiretroviral regimen including at least one of the following: maraviroc, enfuvirtide, raltegravir, etravirine, darunavir/ritonavir or tipranavir/ritonavir. Mutations present in all available genotypic resistance tests were recorded for each patient and then correlated to GSS value, assessed using the last genotypic ribonucleic acid (RNA) resistance test. GSS was studied as predictor of virological treatment outcome by univariate and multivariate logistic regression.

Results: At 48 weeks, undetectable viral load was obtained in $80 \%$ of patients without difference between GSS classes (HIV-RNA median $<50$ copies $/ \mathrm{ml}$ ); $95.8 \%$ of patients with baseline HIV-RNA $<50,000$ copies $/ \mathrm{ml}$ obtained virological suppression ( $\mathrm{p}=0.003) .48$ weeks CD4+ median value was 412 cells $/ \mu 1$ considering GSS1 and 300 cells $/ \mu 1$ for combined GSS2 and GSS3 scores. Data also showed a $>60 \%$ recurrence of specific mutations for NRTI: M41L, M184IV, L210W, T215FY, K219EQ and 75\% for D67N. K103N and Y181CIV mutations for NNRTI persisted in 35\% of cases and their prevalence incresed in parallel with the number of GRTs. About $60 \%$ of tests reported L10FIRVC, M36ILV, M46IL, I54VLAMTS, V82AFTSLI, and L90M mutations in the protease region. 63P mutation was found in a total number of GRTs close to $80 \%$. This percentages, when correlated to GSS, revealed a distinct pattern for most mutations, that showed a greater prevalence for GSS $=2$. Conversely, only NNRTI 181CIV and NRTI 210W showed larger numbers in GSS1 and GSS3.

Conclusions: Single drugs belonging to new antiretroviral classes did not correlate to viroimmunological success for any GSS. High frequency and recurrence over GRTs for specific mutations confirms their key role following the exposure to ARVs classes. A baseline HIV-RNA $<50,000 \mathrm{cp} / \mathrm{ml}$ is a predictor of therapeutic success and a carefully selected HAART based upon the evaluation of GRTs can favorably influence the immunovirologic response.

Keywords: Resistance, HIV, antiretroviral, GSS, mutations.

\section{INTRODUCTION}

Persistent HIV replication in the setting of drug exposure can lead to the appearance of amino acid substitutions that confers resistance to the current antiretroviral (ARV) regimen.

Genotype resistance tests (GRTs) have become the standard of care and are always recommended to guide future regimens following treatment failure [1]. They are

\footnotetext{
*Address correspondence to these authors at Via G.B. Grassi 74 - 20157 Milano, Italy; Tel: +390239042210; E-mail: massi.ortu@gmail.com and Tel: +390239042668; E-mail: stefano.rusconi@unimi.it

${ }^{\S}$ Present Address: Infectious Diseases Unit, Niguarda Hospital, Milan, Italy

${ }^{\natural}$ Present Address: Pio Albergo Trivulzio, via Marostica 8, Milan, Italy
}

based on nucleic acid sequencing of the viral reversetranscriptase and protease genes, and now integrase, and identification of mutations associated with resistance to antiretroviral drugs [1].

Naïve patients represent the "golden standard" for applying GRTs [2], and with a high predictive value usefulness, due to low prevalence of archived mutations [36]. In patients undergoing multiple virological failures, mutation patterns might continuously change and this variability can potentially complicate the interpretation of the genotypic profile. New resistance mutations are constantly developing in patients maintained on virologically ineffective antiretroviral regimens [4].

Consequently, in patients with multiple failures, the extent of drug resistance detected with the last GRT might be underestimated, as several mutations associated with previous treatments might not be detected [5]. In case of 
extended resistance to $\geq 1$ drug classes, neglecting archived mutations (which were not found at the last GRT, but in previous ones) might lead to a rapid failure and even to disease progression [6].

Clinical guidelines now recommend GRT in patients with antiretroviral treatment failure, in drug- naïve patients and in recently infected patients who might have acquired HIV with a drug-resistant virus. The cost of GRT guided therapy, however, is substantial $\left[7^{-10}\right]$ and has prompted a debate about the appropriate use and financing of antiretroviral resistance testing in Europe and the USA. Its costeffectiveness has been documented in a few countries [8].

Some authors $[9,11]$ found that GRT increases projected life-expectancy and quality-adjusted life-expectancy by 2 and 6 weeks, respectively. This increase in quality-adjusted survival is clinically meaningful and comparable to the benefit of elective surgery in patients with symptomatic gallstones (gain in life-expectancy 1.7 months) $\left[{ }^{12}\right]$.

Identification of acquired drug resistance mutations is critical to achieve virologic suppression and improve outcome in patients undergoing multiple virological failures, who present complex mutation profiles [13].

The extent of drug resistance detected can be measured by a score defined as genotypic sensitivity score (GSS); it has been demonstrated to correlate to efficacy $\left[{ }^{12,}{ }^{13}\right]$. Nevertheless, a recent analysis demonstrated that with newest and more potent drugs, the extended resistance profile lacks its association with clinical progression in HIV disease [14]. Physicians caring for subjects with HIV/AIDS have yet to understand the key role of GSS in determining the success of a new antiretroviral regimen that includes the latest generation of drugs or if GSS deserves to be supported by additional tools that are built on new antiretrovirals.

\section{PATIENTS AND METHODS}

\section{Study Patients}

Between March 2008 and April 2008, we selected patients with virological failure starting a new regimen with maraviroc, enfuvirtide, raltegravir, etravirine, darunavir/r or tipranavir/r, alone or in combination among the HIV-1 infected patients followed at Infectious Diseases and Immunopathology Section, University of Milan, Italy. Virological failure was defined by two consecutive HIVRNA values $>50$ copies $/ \mathrm{ml}(\mathrm{cp} / \mathrm{ml})$.

Mutations present in all available genotypic resistance tests were recorded for each patient.

The last genotypic RNA resistance test performed before enrollment was considered to assess the GSS; it was then correlated to viroimmunological data at 6 and 12 months after the beginning of the new regimen.

\section{Virologic and Immunologic Assays}

Genotyping was performed at the Infectious Diseases and Immunopathology Laboratory, University of Milan, Italy and at the "Luigi Sacco" Hospital Central Laboratory, by use of commercial assays based on sequencing of complementary DNA (cDNA) derived from plasma HIV RNA: the TruGene assay (version 2.0; Visible Genetics, Toronto, Canada). It generates a $1.3-\mathrm{kb}$ sequence from the pol region encompassing the entire protease gene and the major part of the reverse-transcriptase gene by bidirectional automated sequencing on the Microgene Clipper (Visible Genetics).

Drug-resistance results were used to guide treatment decisions of patients.

Plasma HIV RNA concentrations were measured by means of a branched DNA (bDNA) assay with a detection limit of $50 \mathrm{cp} / \mathrm{ml}$. Peripheral blood CD4+ lymphocyte counts were performed by use of standard flow cytometry.

\section{Genotypic Susceptibility Scores (GSSs)}

Baseline genotypic mutation patterns from each patient have been correlated to susceptible drugs in the latest ARV regimen, thus obtaining a score (GSS). This GSS was then compared to efficacy data, as virologic response.

A susceptibility score ranging from 0 to 1 was assigned to each drug in the salvage regimen.

We chose to translate definitions of "resistance" and "susceptible or no evidence of resistance" into susceptibility scores of 0 and 1, respectively. Only in cases of molecules not fully active, resistance for the three historical drug classes was assessed using Rega interpretation system (version 8.0.1). The sum of the scores of the individual drugs in the salvage regimen provided the GSS.

The difference between these GSS values is substantial: 0 means that no drugs under the current regimen are actually active against HIV. Likewise, 1, 2 or more indicate the number of active drugs within a regimen.

\section{Statistical Analysis}

The statistical program used for analyses was ANOVA (version 2.0). The differences in the composition of the population were considered statistically significant when the nonparametric Wilcoxon and Mann - Whitney test for continuous variables and the Pearson and Fisher chi - square test for categorical variables showed a probability $<0.05$ compared to the null hypothesis of equal distributions in the groups considered.

\section{RESULTS}

\section{Patients Baseline Characteristics}

A total of 38 patients fulfilling the selection criteria was included into the study. The median age was 39 years (range, 26 - 62 years) and 7 individuals (18\%) were female. HIV risk factors categories were as follows: male homosexuals $(47 \%)$, injection drug users $(32 \%)$, heterosexuals $(20 \%)$ and unknown (1\%). Thirty-eight percent had a previous diagnosis of AIDS (Centers for Disease Control and Prevention class $\mathrm{C}$ criteria $\left.\left[{ }^{15}\right]\right)$.

The median HIV-RNA level was 13,318 copies/ml (range, 369-500,000 copies/ml) and the median CD4+ cell count was 234 cells $/ \mu 1$ (range, 15-1,197 cells/ $\mu 1$ ). Before enrollment patients had been receiving a potent combination regimen for a median of 46 months (range, 18 - 87 months); they had had virologic failure with a median of 2 HAART 
regimens (range 1-7). All patients had been exposed to 3 antiretroviral drug classes.

\section{Salvage Treatments}

Treatments administered after baseline, according to the enrollment criterion, included maraviroc, enfuvirtide, raltegravir, etravirine, darunavir $/ \mathrm{r}$ or tipranavir $/ \mathrm{r}$, alone or in combination.

They consisted of 3 antiretroviral drugs in $69 \%$ and 4 or 5 drugs in $31 \%$ of patients; darunavir was given to 26 patients; etravirine to 13 ; maraviroc to 5 ; raltegravir to 17 ; tipranavir to 2 ; enfuvirtide to 9 .

\section{Virological Response}

After a median follow-up of 48 weeks, 29 patients (76\%) achieved virologic suppression (VS) defined by HIV-RNA $<50$ copies $/ \mathrm{ml}$. VS ranged from $74 \%$ to $78 \%$ without a significant difference when stratified for different GSS (from 1 to 3). This lack of difference between GSS classes was confirmed by a HIV-RNA median value $<50 \mathrm{cp} / \mathrm{ml}$ in any GSS class.

The 24 weeks CD4+ median was $290 / \mu 1$ for GSS1, $299 / \mu 1$ for GSS2 and $367 / \mu 1$ for GSS3. This mild increase from baseline did not significantly correlate to GSS groups ( 1 versus 2 versus 3 or 1 versus 2 plus 3 ). After 48 weeks, the CD4+ median value was $412 / \mu 1$ for GSS 1 and $300 / \mu 1$ for combined GSS2 and GSS3 scores.

Data analysis showed a $>60 \%$ recurrence of specific mutations for NRTI: M41L, M184IV, L210W, T215FY, K219EQ and 75\% for D67N. NNRTI mutations K103N and Y181CIV were identified in the $35 \%$ of GRTs and their prevalence increased in parallel with the number of GRTs. About $60 \%$ of tests reported L10FIRVC, M36ILV, M46IL, I54VLAMTS, V82AFTSLI and L90M mutations in the protease region. L63P mutation was found in a total number of GRTs close to $80 \%$.

As shown in Fig. (1), the prevalence of mutations at week 48 varies when considered in relation to GSS. In detail, mutations are more frequent in absolute for GSS $=2$ versus $\mathrm{GSS}=1$ and $\mathrm{GSS}=3$. This gap is overall equal to $15 \%$ (range $12 \%-17 \%)$.

Conversely, as shown in Fig. (2), only NNRTI mutation Y181CIV and NRTI L210W are less frequent in GSS2 than in GSS1 and GSS3 groups. This gap is quite large, being equal to $50 \%$ (range $49 \%-52 \%$ ).

24 weeks data were similar, although with slightly lower percentages.

Nine patients $(24 \%)$ underwent virological failure at 48 weeks.

Lastly, the period of observation before the start of the study, when all GRTs were performed, amounted to a median of 48 months.

\section{DISCUSSION}

HIV-1 drug resistance is an important factor responsible for failure of antiretroviral treatment. Drug resistance emerges and accumulates over time in treated individuals; nevertheless several new antiretroviral agents with improved activity against resistant viruses have become available during more recent years [16].

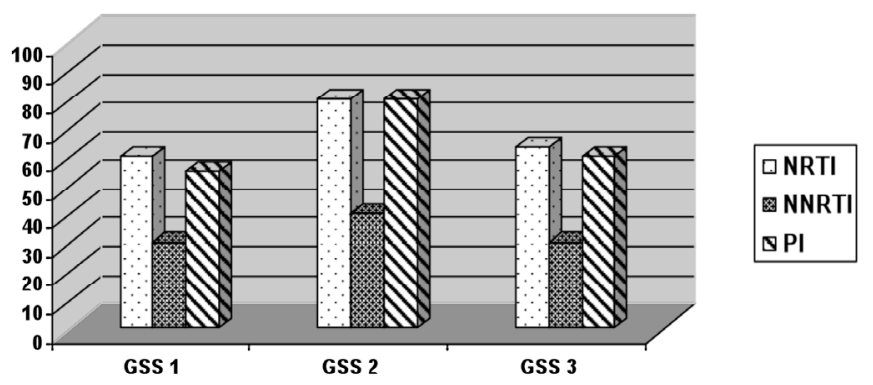

Fig. (1). Frequency of mutations related to different GSS values.

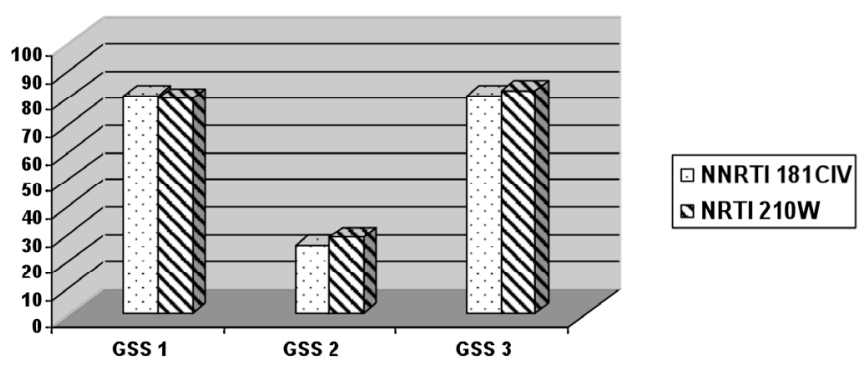

Fig. (2). Different frequency of particular mutations related to GSS values.

Our results at 48 weeks, essentially comparable to 24 weeks, show that starting an antiretroviral regimen with the newest active drugs and drug classes can offer viroimmunological success regardless of GSS1, 2 or 3. Patient groups were homogenous and lack of statistical difference can be easily explained comparing baseline viroimmunologic data, characterized by high viral loads and low CD 4 counts for GSS3 while GSS2 and 1 presented more favorable CD4 counts. This finding is consistent with some studies [17] although other investigations, conducted in the late 1990 decade, support genotypic tests to measure regimen potency as independent predictor of viral response [18].

Six out of the 9 failed patients referred lack of compliance defined as one or more omissions in drug intake per week as clearly related to previous multiple virological failures. One of them died for $P$. jiroveci pneumonia due to persistently low CD4 cell count. Two patients had virological failure probably due to new mutations onset.

On the other hand, GSS, mutations and the newest antiretrovirals were investigated as predictors of virological treatment outcome by univariate and multivariate logistic regression.

Univariate analyses evidenced lack of correlation between specific drugs or mutations and viroimmunological outcome, probably for the intrinsic potency of the newer antiretrovirals.

This might depend on two factors: first, the role of archived drug resistance mutations, still not well understood [19], although a recent paper underlined a better performance of combined current and historical GRTs in identifying patients with a complex resistance profile who may benefit 
from salvage therapies [20]. Undetectable resistances, indeed, would weaken the association between GSS and treatment responses [21].

Secondly, adherence has been shown to be a major factor of virologic failure [10]. This lack of correlation between previous drug resistances and virological outcome could explain the overlap of the results for different values of GSS.

The association emerged between GSS 2 and higher frequency of mutations could be explained by a better compliance of this category of patients. A great deal of adherence would cause a wider and longer drug pressure by which viral strains are forced to maintain key mutations. This furtherly confirms the key role of mutations following the exposure to certain ARVs.

According to the literature, we should stress that the finding of a median baseline HIV-RNA $<50,000 \mathrm{cp} / \mathrm{ml}$ is more likely associated to virological success.

It was not possible to carry out multivariate analysis due to small number of patients, which is a major limitation of our study. Preliminary assessment on the power of the study ruled that a sufficient number of patients would have been equal to 80 . Failed patients in our Clinic were not so many and multicenter study could not have been possible, worth the change in the methods.

Our results evidence that a carefully selected HAART based upon the evaluation of GRTs can favorably influence the immunovirologic response at 24 and most significantly at 48 weeks.

Only a longer period of observation will confirm the durability of the new ARV regimens in relation to GSS 1, 2 or 3 . Nevertheless these data should be confirmed in larger cohorts.

\section{SUMMARY}

The frequency and the recurrence across genotypic resistance tests (GRTs) of the major mutations correlate to genotypic sensitivity score value (GSS); therefore, a HAART selected on the basis of a careful evaluation of GRTs can favorably influence the 48 weeks immunovirologic response.

\section{ACKNOWLEDGEMENTS}

This work was made by the authors listed above, according to our original idea and plan without involving any external consulent, specialist or writer.

It did not receive any kind of funding, internal or external; data have been generated as part of the routine work of our Department.

No ongoing financial support for any of the authors is present.

\section{TRANSPARENCY DECLARATIONS}

None to declare; my co-authors and I do not have any financial conflict interests.

\section{ETHICAL DECLARATIONS}

Since we performed an observational study, no ethics committee approval or written informed consent from the participants were required.

\section{REFERENCES}

[1] Panel on Antiretroviral Guidelines for Adults and Adolescents. Guidelines for the use of antiretroviral agents in HIV-1-infected adults and adolescents. US Department of Health and Human Services. January 2011. Available from http://www.aidsinfo.nih.gov

[2] Shafer RW, Jung DR, Betts BJ, Xi Y, Gonzales MJ. Human immunodeficiency virus reverse transcriptase and protease sequence database. Nucleic Acids Res 2000; 28: 346-8.

[3] Sax PE, Islam R, Walensky RP, et al. Should resistance testing be performed for treatment-naive HIV-infected patients? A costeffectiveness analysis. Clin Infect Dis 2005; 41: 1316-23.

[4] Harrigan PR, Cote HC. Clinical utility of testing human immunodeficiency virus for drug resistance. Clin Infect Dis 2000; 30 Suppl 2: S117-S122.

[5] Clavel F, Hance AJ. HIV drug resistance. N Engl J Med 2004; 350 . 1023-35.

[6] Cozzi-Lepri A, Phillips AN, Ruiz L, et al. Evolution of drug resistance in $\mathrm{HIV}$-infected patients remaining on a virologically failing combination antiretroviral therapy regimen. AIDS 2007; 21 : 721-32.

[7] Yerly S, Fagard C, Günthard HF, Hirschel B, Perrin L, Swiss HIV Cohort Study. Drug resistance mutations during structured treatment interruptions. Antivir Ther 2003; 8: 411-5.

[8] Harrigan PR, Wynhoven B, Brumme ZL, et al. HIV-1 drug resistance: degree of underestimation by a cross-section. $\mathrm{J}$ Infect Dis 2005; 191: 1325-30.

[9] Simcock M, Sendi P, Ledergerber B, et al. A longitudinal analysis of health care costs after treatment optimisation following genotypic antiretroviral resistance testing: does resistance testing pay off? Antivir Ther 2006; 11: 305-14.

[10] Weinstein MC, Goldie SJ, Losina E, et al. Use of genotypic resistance testing to guide HIV therapy: clinical impact and costeffectiveness. Ann Intern Med 2001; 134: 440-50.

[11] Sendi P, Gunthard HF, Simcock M, et al. Cost-effectiveness of genotypic antiretroviral resistance testing in HIV-infected patients with treatment failure. PLoS One 2007; 2: e173.

[12] Castor D, Vlahov D, Hoover DR, et al. The relationship between genotypic sensitivity score and treatment outcomes in late stage HIV disease after supervised HAART. J Med Virol 2009; 81: 1323-35.

[13] Anderson JA, Jiang H, Ding X, et al. Genotypic Susceptibility Scores and HIV Type 1 RNA responses in treatment-experienced subjects with HIV type 1 infection. AIDS Res Hum Retroviruses 2008; 24: 685-94.

[14] Zaccarelli M, Lorenzini P, Marconi P, et al. Is extended resistance to the historical antiretroviral drugs \& drug classes still a risk factor for HIV progression? Tenth International Congress on Drug Therapy in HIV Infection, 7-11 November 2010, Glasgow, UK; P138.

[15] Centers for Disease Control and Prevention. 1993 Revised classification system for HIV infection and expanded surveillance case definition for AIDS among adolescents and adults. MMWR Morb Mortal Wkly Rep 1992; 41(RR-17): 1-19.

[16] Martinez-Cajas JL, Wainberg A. Protease inhibitor resistance in HIV-infected patients: molecular and clinical perspectives. Antiviral Res 2007; 76: 203-21.

[17] Mazzotta F, Lo Caputo S, Torti C, et al. Real versus virtual phenotype to guide treatment in heavily pretreated patients: 48week follow-up of the Genotipo-Fenotipo di Resistenza (GenPheRex) trial. J Acquir Immune Defic Syndr 2003; 32: 268 80 .

[18] DeGruttola V, Dix L, D'Aquila R, et al. The relation between baseline HIV drug resistance and response to antiretroviral therapy: re-analysis of retrospective and prospective studies using a standardized data analysis plan. Antivir Ther 2000; 5: 41-8. 
[19] Delaugerre C, Teglas JP, Treluyer JM, et al. Predictive factors of virologic success in HIV-1-infected children treated with lopinavir/ritonavir. J Acquir Immune Defic Syndr 2004; 37(2): $1269-75$.
[20] Zaccarelli M, Lorenzini $\mathrm{P}$, Ceccherini-Silberstein $\mathrm{F}$, et al. Historical resistance profile helps to predict salvage failure. Antivir Ther 2008; 14: 285-91.

[21] Peuchant O, Thiébaut R, Capdepont S, et al. Transmission of HIV1 minority-resistant variants and response to first-line antiretroviral therapy AIDS 2008; 22: 1417-23. 